\title{
Boundary-Layer Effects on the Reflection of Sound Waves and Weak Shock Waves at Shallow Incidence
}

By Arthur F. Messiter, Department of Aerospace Engineering, The University of Michigan, Ann Arbor, Michigan, USA

This paper is dedicated to Nicholas Rott, in appreciation for his helping to introduce me to aerodynamics, and in honor of his sixtieth birthday.

\section{Introduction}

If a plane acoustic wave is incident upon an infinite plane wall, the reflected wave has smaller amplitude than the incident wave because of the time-dependent boundary layer along the wall. Zogg [1] has noted that the boundary-layer correction to the potential for the reflected wave is singular as the direction of propagation becomes parallel to the wall. The largest correction term becomes proportional to the ratio $k \delta / \theta$, where $\delta$ is the boundary-layer thickness, $1 / k$ is the wave length of the incident wave, and $\theta$ is the angle, for the incident wave, between the wave front and the normal to the wall. Zogg concluded that his solution is valid only if this ratio is small. By retaining nonlinear terms in the potential equation and transforming to a coordinate system moving with the wave, it is shown here that for small values of $\theta$ the boundary-layer effect can be described in terms of a transonic-flow problem. The resulting approximate differential equation is linear with variable coefficients, and describes small perturbations about a prescribed sound wave moving parallel to the wall. The revised approximation becomes necessary when the angle $\theta$ is of the same order as the square root of the nondimensional wave amplitude.

A similar nonuniformity appears if a weak plane shock wave is reflected from an infinite wall. The linear-theory prediction of the boundary-layer contribution to the potential in the flow behind the reflected wave becomes large as the angle $\theta_{I}$ between the incident wave and the normal to the wall becomes small. In a coordinate system moving with the wave the flow is steady, and when $\theta_{I}$ is small the shock-wave reflection can be described by the transonic approximation to the shock-polar equation. The position of the reflected wave is then known in a first approximation and so, for values of $\theta_{I}$ larger than the minimum value for which regular reflection is possible, the boundary-layer correction is derived quite easily. The modified approximation is required when the angle $\theta_{l}$ is of the order of the square root of the nondimensional shock-wave strength. 


\section{Reflection of a Sound Wave at Shallow Incidence}

The reflection of an incident plane acoustic wave from an infinite plane wall $y=0$ is described by a potential

$$
\phi(x, y, t)=i \varepsilon \frac{a_{0}}{k} e^{i\left(\omega t-k_{x} x\right)} \cos k_{y} y
$$

where $x$ is measured along the wall; $a_{0}$ is the sound speed in the undisturbed gas; $\varepsilon \ll 1$ is the dimensionless amplitude of the incident wave; $k_{y} / k_{x}=\tan \theta$; and $k^{2}=k_{x}^{2}+k_{y}^{2}$ $=\omega^{2} / a_{0}^{2}$. The result (1) is a solution to the wave equation having a prescribed form for the incident wave and satisfying the boundary condition $\phi_{y}=0$ at $y=0$. At $y=0$,

$$
\phi_{x}(x, 0, t)=\varepsilon a_{0} \frac{k_{x}}{k} e^{i\left(\omega t-k_{x} x\right)}
$$

In the boundary layer

$$
\rho_{0} u_{t}+p_{x}=\mu_{0} u_{y y}, \quad p_{y}=0
$$

where the pressure is found from (1) using $p-p_{0}=-\rho_{0} \phi_{t} ; p_{0}, \rho_{0}$, and $\mu_{0}$ are the pressure, density, and viscosity coefficient in the undisturbed gas; and $u$ is the $x$ component of velocity. The boundary-layer approximation requires $\omega v_{0} / a_{0}^{2} \ll 1$, where $v_{0}=\mu_{0} / \rho_{0}$; that is, we take $k \delta \ll 1$, where $\delta=\left(2 v_{0} / \omega\right)^{1 / 2}$. The solution of (3) given by

$$
u=\varepsilon a_{0} \frac{k_{x}}{k} e^{i\left(\omega t-k_{x} x\right)}\left(1-e^{-(1+i) y / \delta}\right)
$$

satisfies the boundary condition $u=0$ at $y=0$ and approaches the value of $\phi_{x}$ given by (2) as $y / \delta \rightarrow \infty$. The temperature profile is calculated using the energy equation; it is assumed that the wall temperature remains equal to the temperature in the undisturbed gas. The density is found from the equation of state, and it then follows from the continuity equation that the boundary-layer contribution to the $y$-component of velocity as $y / \delta \rightarrow \infty$ is

$$
\Delta v=-\frac{1}{2}(1+i)\left(\frac{\gamma-1}{\sigma^{1 / 2}}+\frac{k_{x}^{2}}{k^{2}}\right) \varepsilon k \delta a_{0} e^{i\left(\omega t-k_{x} x\right)}
$$

where $\gamma$ is the ratio of specific heats, $a_{0}^{2}=\gamma p_{0} / \rho_{0}$, and $\sigma$ is the Prandtl number; this expression is consistent with the result given by Rott [2].

A second-order term can now be added to the external potential flow [1], in the form of a correction to the reflected wave satisfying the boundary condition that 
$\phi_{y}(x, 0, t)$ equals the value of $\Delta v$ given by (5). It is found that

$$
\begin{aligned}
\phi(x, y, t)= & i \varepsilon \frac{a_{0}}{k} e^{i\left(\omega t-k_{x} x\right)} \cos k_{y} y \\
& +\frac{1}{2}(1-i)\left(\frac{\gamma-1}{\sigma^{1 / 2}}+\frac{k_{x}^{2}}{k^{2}}\right) \varepsilon a_{0} k \delta \frac{1}{k_{y}} e^{i\left(\omega t-k_{x} x-k_{y} y\right)}
\end{aligned}
$$

The second term in (6) is small in comparison with the first term if $k \delta \ll \theta$. However, this may not be a sufficient condition for the validity of (6), and the nature of the proper approximation for small $\theta$ is not obvious. Resolution of these questions requires a nonlinear potential equation and discussion of the relative sizes of the three small parameters $\varepsilon, k \delta$, and $\theta$.

For irrotational isentropic flow the continuity and Bernoulli equations are

$$
\begin{aligned}
& \rho_{t}+\rho \nabla^{2} \phi+\nabla \phi \cdot \nabla \rho=0 \\
& \phi_{t}+\frac{1}{2} q^{2}+\frac{a_{0}^{2}}{\gamma-1}\left(\frac{\rho}{\rho_{0}}\right)^{\gamma-1}=\frac{a_{0}^{2}}{\gamma-1}
\end{aligned}
$$

A first approximation gives $a_{0}^{2}\left(\rho-\rho_{0}\right)=-\rho_{0} \phi_{i}$ and $\phi_{t z}=a_{0}^{2} \phi_{x x}$. An equation for $\phi$ which is accurate to second order is then

$$
a_{0}^{2} \nabla^{2} \phi-\phi_{t t}=2 \nabla \phi \cdot \nabla \phi_{t}+(\gamma-1) \phi_{t} \nabla^{2} \phi
$$

Substitution of the solution (6) in the right-hand side of (9) gives terms of order $\varepsilon^{2} a_{0}^{2} \omega$ and $\varepsilon^{2} a_{0}^{2} \omega(k \delta / \theta)$. Since the terms in $a_{0}^{2} \phi_{y y}$ on the left-hand side are of order $\varepsilon a_{0}^{2} \omega \theta^{2}$ and $\varepsilon a_{0}^{2} \omega \theta^{2}(k \delta / \theta)$, there is an implication, which will be examined below, that the approximate solution (6) may require $\theta \gg \varepsilon^{1 / 2}$. If $\theta=O\left(\varepsilon^{1 / 2}\right)$, the order of magnitude of the boundary-layer correction, but not its functional form, can be found by replacing $\theta$ with $\varepsilon^{1 / 2}$ in (6). The conjecture then is that this correction is of order $\varepsilon^{1 / 2} a_{0} \delta$ and does not become larger if $\theta / \varepsilon^{1 / 2} \rightarrow 0$. Only the case $k \delta \ll \varepsilon^{1 / 2}$ will be considered, since this case corresponds to a useful range of numerical values and also leads to certain simplifications. The condition $\theta \gg \varepsilon^{1 / 2}$ for the validity of (6) is then a stronger requirement than the condition $\theta \gg k \delta$ originally suggested.

The nature of the nonuniformity for small $\theta$ is perhaps made more clear if we consider small perturbations about a small-amplitude wave traveling parallel to the wall. A coordinate system moving in the positive $x$-direction at speed $a_{0} / \cos \theta$ is introduced by the transformation

$$
\tilde{x}=k\left(x \cos \theta-a_{0} t\right)
$$

where $k=\omega / a_{0}$, as before. The potential equation (9) can be rewritten for $\theta \ll 1$ with the nonlinear terms simplified by use of the approximation $\phi_{t} \sim-a_{0} \phi_{x}$. The result is, in a temporary mixed notation,

$$
-k^{2} a_{0}^{2} \theta^{2} \phi_{\tilde{x} \tilde{x}}+a_{0}^{2} \phi_{y y}+2 a_{0} k \phi_{\tilde{x} t}=-a_{0} k^{3}(\gamma+1) \phi_{\tilde{x}} \phi_{\tilde{x} \tilde{x}}+\cdots
$$


and it is easily shown that all omitted terms are necessarily smaller than those retained. The signs here appear unfamiliar because the flow is from right to left; if $\tilde{x}$ were replaced by $-\tilde{x}$, the usual signs would be obtained. In (11), $\partial / \partial t$ implies differentiation with $\tilde{x}$ held fixed, and in this system it is expected that the solution will change only slowly with $t$. For the largest term in $\phi$, the part corresponding to an incoming wave is considered to be prescribed, and the condition $\phi_{y}=0$ at $y=0$ is to be satisfied. For the second term, there is no incoming wave and the $y$-derivative has the value given by (5) as $y \rightarrow 0$. If only the first two terms in (11) are retained, the solution (6) is obtained, with $\sin \theta$ replaced by $\theta$, etc.

Motivated in part by the considerations discussed above and in part by the anticipated nature of the results, we try the following asymptotic expansions of the potential in the limit as $\varepsilon \rightarrow 0$ and $\theta \rightarrow 0$ with $\theta^{2} / \varepsilon$ fixed:

$$
\begin{aligned}
& \phi=\left(\varepsilon a_{0} / k\right) \phi_{0}(\tilde{x}, \tilde{y}, \tilde{t})+\varepsilon^{1 / 2} a_{0} \delta \phi_{1}(\tilde{x}, \tilde{y}, \tilde{t})+\cdots \\
& \tilde{y}=\varepsilon^{1 / 2} k y, \quad \tilde{t}=\varepsilon \omega t
\end{aligned}
$$

The characteristic time is taken as $(\varepsilon \omega)^{-1}$ because in the moving coordinate system the motion is nearly steady, but the nonlinear wave steepening is proportional to the square of the amplitude and the resulting change in waveform is no longer negligible when the time becomes of this order. When this assumed form of solution is substituted in (11), the largest terms are found to give

$$
\left(\theta^{2} / \varepsilon\right) \phi_{0 \tilde{x} \tilde{x}}-\phi_{0 \tilde{y} \tilde{y}}=2 \phi_{0 \tilde{x} \tilde{i}}+(\gamma+1) \phi_{0 \tilde{x}} \phi_{0 \tilde{x} \tilde{x}}
$$

This is the unsteady form of the transonic small-disturbance equation. If $\theta^{2} / \varepsilon \rightarrow \infty$, then $\tilde{y}=O\left(\varepsilon^{1 / 2} / \theta\right)$ and the terms on the left-hand side are dominant; the solution satisfying proper initial and boundary conditions again gives (1). If $\theta^{2} / \varepsilon \rightarrow 0$, the largest terms are those on the right-hand side, and the resulting equation describes a plane wave traveling in the positive $\tilde{x}$-direction with $d \phi_{0 \tilde{x}} / d \tilde{t}=0$ along characteristics $d \tilde{x} / d \tilde{t}$ $\sim \frac{1}{2}(\gamma+1) \phi_{0 \tilde{x}}$.

The differential equation for the second approximation is

$$
\left(\theta^{2} / \varepsilon\right) \phi_{1 \tilde{x} \tilde{x}}-\phi_{1 \tilde{y} \tilde{y}}=2 \phi_{1 \tilde{x} \tilde{t}}+(\gamma+1)\left(\phi_{0 \tilde{x}} \phi_{1 \tilde{x}}\right)_{\tilde{x}}
$$

This is the equation which would have to be studied if the boundary-layer correction were to be calculated for $\theta=O\left(\varepsilon^{1 / 2}\right)$. For $\theta^{2} / \varepsilon \rightarrow \infty$, a solution of (15) satisfying the boundary condition obtained from (5) again leads to the correction term in (6). For $\theta^{2} / \varepsilon=O(1)$, the equation is linear with variable coefficients which depend on the solution for $\phi_{0}$. The boundary condition at $\tilde{y}=0$ would again be obtained from (5), and some kind of initial condition would be required. For example, one might consider a semi-infinite plate $y=0, x>0$ with the incoming wave prescribed as sinusoidal at $x$ $=0$. For $\theta^{2} / \varepsilon \rightarrow 0$, the first term in (15) disappears, and the resulting equation describes the boundary-layer perturbations on a wave traveling parallel to the wall. Solutions to this problem are expected to exist, and so it seems that solutions for $\phi_{1}$ must remain bounded as $\theta^{2} / \varepsilon \rightarrow 0$. Thus for small $\theta$ the boundary-layer correction to $\phi$ appears to 
become no larger than $O\left(\varepsilon^{1 / 2} a_{0} \delta\right)$ and the anticipated restriction $\theta \ll \varepsilon^{1 / 2}$ for the validity of (6) appears to be correct.

\section{Reflection of a Weak Nearly Normal Shock Wave}

A plane shock wave moving at speed $c_{s}$, into a gas initially at rest and having uniform properties, gives a velocity $\varepsilon a_{0}$ to the gas, where for weak shock waves $\varepsilon \ll 1$ and $c_{s} / a_{0}=1+\frac{1}{4}(\gamma+1) \varepsilon+\cdots$. If the shock wave is reflected from an infinite plane wall, at an angle $\theta_{I}$ from the normal, the reflected shock wave lies at an angle $\theta_{I}+O(\varepsilon)$ from the normal, and behind the reflected shock wave the flow is parallel to the wall at speed $\Delta u=2 \varepsilon a_{0} \cos \theta_{I}+\cdots$. In a coordinate system moving parallel to the wall at a speed $u_{\infty}=c_{s} / \cos \theta_{I}$, the flow is steady. Coordinates $x$ and $y$ will be measured along and normal to the wall, with $x$ positive to the right. We consider a shock wave moving to the left, so that the steady flow now is in the positive $x$-direction, from left to right. The velocity components $u_{1}$ and $v_{1}$, in the $x$ and $y$ directions respectively, in the region behind the incident shock wave are

$$
\begin{aligned}
& u_{1}=\frac{c_{s}}{\cos \theta_{I}}-\varepsilon a_{0} \cos \theta_{I} \\
& v_{1}=-\varepsilon a_{0} \sin \theta_{I}
\end{aligned}
$$

The velocity $u_{2}$ behind the reflected shock wave is

$$
u_{2}=u_{\infty}-\Delta u=\frac{c_{s}}{\cos \theta_{I}}-2 \varepsilon a_{0} \cos \theta_{I}+\cdots
$$

The boundary layer behind the reflected shock wave, described by linearized equations, has velocity profile

$$
u-u_{\infty}=-(\Delta u) \operatorname{erf}(y / \delta)
$$

where $u_{\infty}-\Delta u \leqslant u \leqslant u_{\infty}$ and $\delta=\left(4 v_{0} x / u_{\infty}\right)^{1 / 2}$, for $x>0$. If the wall and undisturbed gas temperatures are equal, the continuity equation gives a velocity component normal to the wall, as $y / \delta \rightarrow \infty$,

$$
v \sim-\frac{1+(\gamma-1) \sigma^{-1 / 2} M_{\infty}^{2}}{\pi^{1 / 2}}(\Delta u)\left(\frac{v_{0}}{u_{\infty} x}\right)^{1 / 2}
$$

In the external flow behind the reflected shock wave, the small displacement effect of the boundary layer gives an additional term in the potential, so that now

$$
\begin{aligned}
\phi= & u_{2} x+2 \frac{1+(\gamma-1) \sigma^{-1 / 2} M_{\infty}^{2}}{\pi^{1 / 2}\left(M_{2}^{2}-1\right)^{1 / 2}} \\
& \times(\Delta u)\left(\frac{v_{0}}{u_{\infty}}\right)^{1 / 2}\left\{x-\left(M_{2}^{2}-1\right)^{1 / 2} y\right\}^{1 / 2}
\end{aligned}
$$


where $M_{2}=u_{2} / a_{2}$ is the Mach number in the external flow behind the reflected shock wave, for $v_{0} \rightarrow 0$, and is approximately equal to $M_{\infty}=u_{\infty} / a_{0}$. In the combination $M_{2}^{2}$ -1 above, $M_{2}$ is used somewhat arbitrarily in place of $M_{\infty}$. Since $\varepsilon \ll 1$,

$$
\begin{aligned}
\left(M_{2}^{2}-1\right)^{1 / 2}= & \left(M_{\infty}^{2}-1\right)^{1 / 2}\left\{1+\left(1+\frac{\gamma-1}{2} M_{\infty}^{2}\right)\right. \\
& \left.\times \frac{M_{\infty}^{2}}{M_{\infty}^{2}-1} \frac{\Delta u}{u_{\infty}}+\cdots\right\}
\end{aligned}
$$

Therefore the boundary-layer correction to $\phi_{x}$ as found from (21) is proportional to $\left(M_{\infty}^{2}-1\right)^{-1 / 2}\left(u_{\infty} x / v_{0}\right)^{-1 / 2} \Delta u$. Since the shock-wave thickness is of order $\varepsilon^{-1} v_{0} / u_{\infty}$, the correction term remains small in comparison with $\Delta u$ for values of $x$ which are large in comparison with the shock-wave thickness.

Using (21), we can expand the slope $\tan (\theta+\mu)$ of a downstream-running characteristic, where $\mu=\sin ^{-1}(1 / M)$ and $\theta$ is now the flow deflection angle. A characteristic $\xi=$ constant is given by

$$
\begin{aligned}
y= & (x-\xi)\left\{\frac{1}{\left(M_{2}^{2}-1\right)^{1 / 2}}-\frac{\gamma+1}{2} \frac{M_{\infty}^{4}}{\left(M_{2}^{2}-1\right)^{2}}\right. \\
& \left.\times \frac{1+(\gamma-1) \sigma^{-1 / 2} M_{\infty}^{2}}{\pi^{1 / 2}} \frac{\Delta u}{u_{\infty}}\left(\frac{v_{0}}{u_{\infty} \xi}\right)^{1 / 2}+\cdots\right\}
\end{aligned}
$$

where $\xi$ is defined by setting $x=\xi$ at $y=0$. Since a weak shock wave bisects the angle between upstream and downstream characteristics, the angle $\theta_{R}$ between the reflected shock wave and the normal to the wall is, approximately, $\theta_{R}=\frac{1}{2} \pi-\frac{1}{2}\left(\mu_{2}+\mu_{1}+\theta_{1}\right)$, where the subscripts 1 and 2 again refer to conditions behind the incident and reflected shock waves respectively. A relation between $\theta_{1}$ and $\theta_{l}$ is found by setting $v_{1} / u_{\infty} \sim \theta_{1}$ in (17). Since $\mu_{1}-\mu_{\infty}$ and $\mu_{2}-\mu_{1}$ are approximately proportional to $\theta_{1}$, we can express $\theta_{R}$ in terms of $\theta_{I}$. If a boundary-layer correction is added,

$$
\begin{aligned}
\theta_{R}=\theta_{I} & +\left(1-\frac{1+\frac{1}{2}(\gamma-1) \sec ^{2} \theta_{I}}{\tan ^{2} \theta_{I}}\right) \varepsilon \sin \theta_{I} \cos \theta_{I}+\cdots \\
& +\frac{\gamma+1}{4} \frac{M_{\infty}^{2}}{M_{\infty}^{2}-1} \frac{1+(\gamma-1) \sigma^{-1 / 2} M_{\infty}^{2}}{\pi^{1 / 2}} \frac{\Delta u}{u_{\infty}}\left(\frac{v_{0}}{u_{\infty} \xi}\right)^{1 / 2}+\cdots
\end{aligned}
$$

Since $M_{\infty}^{2}-1 \sim\left(\sin ^{2} \theta_{I}+\frac{1}{2}(\gamma+1) \varepsilon+\cdots\right) / \cos ^{2} \theta_{l}$, it is evident from (22) and (24) that neglected terms in the approximations $M_{2}^{2}-1 \sim M_{\infty}^{2}-1$ and $\theta_{R} \sim \theta_{I}$ will remain small in comparison with the first terms only if $\theta_{I} \ll \varepsilon^{1 / 2}$. At transonic speeds the velocity components behind the incident shock wave are found using (16) and (17) to be

$$
\begin{aligned}
& u_{1} / a^{*}=1-\frac{1}{2} \varepsilon+\theta_{I}^{2} /(\gamma+1)+\cdots \\
& v_{1} / a^{*}=-\varepsilon \theta_{I}+\cdots
\end{aligned}
$$


The approximate shock-wave slope and shock-polar equation for the reflected shock wave are given by

$$
\begin{gathered}
\theta_{R}=\frac{-v_{1}}{u_{1}-u_{2}} \\
\left(\frac{v_{1}}{a^{*}}\right)^{2}=\frac{\gamma+1}{2}\left\{\left(\frac{u_{1}}{a^{*}}-1\right)-\left(\frac{u_{2}}{a^{*}}-1\right)\right\}^{2} \\
\times\left\{\left(\frac{u_{1}}{a^{*}}-1\right)+\left(\frac{u_{2}}{a^{*}}-1\right)\right\}
\end{gathered}
$$

Thus $u_{2}$ and $\theta_{R}$ are expressed implicitly in terms of $\theta_{1}$ and $\varepsilon$. In the special case for which the gas velocity is sonic behind the reflected shock, $M_{2}=1$ and $u_{2}=a^{*}$, and we find

$$
\frac{2 \theta_{I}^{2}}{(\gamma+1) \varepsilon}=\frac{1}{8}\left(\frac{2 \theta_{I}^{2}}{(\gamma+1) \varepsilon}-1\right)^{3}
$$

Then $2 \theta_{1}^{2} /(\gamma+1) \varepsilon \approx 4.24$. For any given value of $u_{1}$, there is also a value of $u_{2}$ for which the right-hand side of (28) is a maximum, corresponding to a maximum flow deflection angle across the reflected shock wave. If this condition is combined with (25), (26), and (28), it is found that the minimum value of the angle $\theta_{l}$ for which regular reflection is possible is given by

$$
\frac{2 \theta_{I}^{2}}{(\gamma+1) \varepsilon}=\frac{4}{27}\left(\frac{2 \theta_{I}^{2}}{(\gamma+1) \varepsilon}-1\right)^{3}
$$

and $2 \theta_{I}^{2} /(\gamma+1) \varepsilon=4$.

To calculate the boundary-layer displacement effect for $\theta_{I}=O\left(\varepsilon^{1 / 2}\right)$, we first introduce small perturbations in the shock-wave relations. If $u_{2}^{(1)}$ and $v_{2}^{(1)}$ are the perturbations in velocity components downstream of the reflected shock wave and $\theta_{R}^{(1)}$ is the perturbation in the angle between the reflected shock wave and the normal to the wall, we find at the shock wave

$$
\begin{aligned}
& \frac{v_{2}^{(1)}}{a^{*}}=-\frac{1}{2}\left(\frac{\gamma+1}{2}\right)^{1 / 2} \frac{\left(\frac{u_{1}}{a^{*}}-1\right)+3\left(\frac{u_{2}}{a^{*}}-1\right)}{\left\{\left(\frac{u_{1}}{a^{*}}-1\right)+\left(\frac{u_{2}}{a^{*}}-1\right)\right\}^{1 / 2}} \frac{u_{2}^{(1)}}{a^{*}} \\
& \frac{\theta_{R}^{(1)}}{\theta_{R}}=-\frac{v_{2}^{(1)}}{v_{1}}+\frac{u_{2}^{(1)}}{u_{1}-u_{2}}
\end{aligned}
$$

where $y=x / \theta_{R}$ has been used as the first approximation to the shock-wave position. 
For the flow behind the shock wave, the differential equation for the perturbation potential is

$$
\left(M_{2}^{2}-1\right) \phi_{2 x x}^{(1)}=\phi_{2 y y}^{(1)}
$$

where now $M_{2}^{2}-1 \sim(\gamma+1)\left(1-u_{2} / a^{*}\right)$. The approximation used earlier for $\theta_{I}^{2} \gg \varepsilon$ required only outgoing waves, but for $\theta_{I}^{2}=O(\varepsilon)$ the shock-wave relations require both families of waves. Using (20), the form of solution is

$$
\phi_{2}^{(1)}=\alpha(x-\lambda y)^{1 / 2}+\beta(x+\lambda y)^{1 / 2}
$$

where $\lambda=\left(M_{2}^{2}-1\right)^{1 / 2}$, and the values of the constants $\alpha$ and $\beta$ are found from (20) and (31). If $M_{2}<1$, but $\theta_{I}$ is still large enough to permit regular reflections, the differential equation for $\phi_{2}^{(1)}$ is still (33) and the form of solution is

$$
\begin{aligned}
& \phi_{2}^{(1)}=A r^{1 / 2} \cos \frac{1}{2} \vartheta+B r^{1 / 2} \sin \frac{1}{2} \vartheta \\
& r^{2}=x^{2}+\lambda^{2} y^{2}, \quad \vartheta=\tan ^{-1} \frac{\lambda y}{x}
\end{aligned}
$$

where now $\lambda^{2}=1-M_{2}^{2}$. The constants $A$ and $B$ are found from (20) and (31).

It is found from the solutions (34) and (35) that the perturbation velocity $u_{2}^{(1)}$ is of order $u_{\infty} \varepsilon^{1 / 2}\left(u_{\infty} x / v_{0}\right)^{-1 / 2}$ and does not grow indefinitely large as $M_{2} \rightarrow 1$, as would typically be the case in a small-perturbation solution. For $\left|M_{2}^{2}-1\right| \ll \varepsilon$ the characteristic length in the $y$ direction is the distance $x / \theta_{R}$ from the wall to the shock wave, which is small in comparison with the characteristic length $O(x / \lambda)$ obtained if the two terms in the differential equation are assumed to be of the same order. Thus for $\left|M_{2}^{2}-1\right| \ll \varepsilon$ the proper scaling for the $y$-coordinate involves $\theta_{R}$ rather than $\lambda$, and when written in proper variables the limiting form of the differential equation contains a single term:

$$
\phi_{2 y y}^{(1)}=0
$$

The solution $\phi_{2}^{(1)}=f(x) y+g(x)$ is required to satisfy the boundary conditions at $y=0$ and at the shock wave, and is found to agree with the solutions (34) and (35) evaluated for $M_{2} \rightarrow 1$.

Finally, if $\left(M_{2}^{2}-1\right) / \varepsilon \rightarrow 0$ and also $x \rightarrow 0$, the boundary-layer correction $u_{2}^{(1)}$ will be large in comparison with $\left|u_{2}-a^{*}\right|$ if $\varepsilon a x / v \ll \varepsilon^{2} /\left(M_{2}^{2}-1\right)^{2}$ but remains small in comparison with $a^{*}$ provided that $\varepsilon a_{0} x / v \gg 1$. Thus the boundary-layer correction is calculated as a simple displacement effect as long as the distance from the shock wave is much larger than the shock-wave thickness. For $x=O\left(\varepsilon^{-1} v_{0} / a_{0}\right)$ the problem becomes an interaction problem of the type described by Sichel [3] for a normal shock wave. The boundary-layer thickness here is of order $\varepsilon^{-1 / 2} v_{0} / a_{0}$, smaller than $\varepsilon^{-1} v_{0} / a_{0}$, and so the boundary layer can still be considered thin, but the pressure now appears in the momentum equation and is not known in advance. In an outer region where $x$ $=O\left(\varepsilon^{-1} v_{0} / a\right)$ and $y=O\left(\varepsilon^{-3 / 2} v_{0} / a\right)$, the correct approximate equations describe a two-dimensional shock structure with $v / a^{*}=O\left(\varepsilon^{3 / 2}\right)$. 


\title{
References
}

[1] H. ZoGG, Diffraktion von schwachen Stössen an schlanken Körpern und der Einfluss der Grenzschicht, Diss. ETH Zürich Nr. 5496 (1975).

[2] N. RotT, The Influence of Heat Conduction on Acoustic Streaming, Z. angew. Math. Phys. 25, 417 (1974).

[3] M. SICHEL, Leading Edge of a Shock-Induced Boundary Layer, Phys. Fluids 5, 1168 (1962).

\begin{abstract}
When either a plane acoustic wave or a weak shock wave is incident upon an infinite wall, the boundarylayer correction to the reflected wave becomes large if the angle between the wave front and the normal to the wall approaches zero. It is shown that transformation to moving coordinates leads to transonic flow problems whose solution permits removal of the singular behavior in each of these cases.
\end{abstract}

\section{Zusammenfassung}

Wenn eine ebene Schallwelle oder ein schwacher Stoss so auf eine Wand auftriff, dass der Winkel zwischen der Wellenfront und der Wandnormalen gegen Null geht, so wird die Korrektur der reflektierten Welle wegen der Wandgrenzschicht gross. In der vorliegenden Arbeit wird gezeigt, dass eine Transformation auf bewegte Koordinaten auf ein transonisches Problem führt, dessen Lösung die Singularität in beiden Fällen vermeidet.

(Received: April 25, 1977) 